Article

\title{
Preparation and Chromatographic Application of $\beta$-Cyclodextrin Molecularly Imprinted Microspheres for Paeoniflorin
}

\author{
Wei Zhang ${ }^{1}$, Bofeng Wei ${ }^{2}$, Shoujiang $\mathrm{Li}^{2}$, Yueming Wang ${ }^{2}$ and Shaoyan Wang ${ }^{1,2, *}$ \\ 1 School of Chemical Engineering, University of Science and Technology, Anshan 114051, Liaoning, China; \\ askdzw@ustl.edu.cn or askdzw@163.com \\ 2 Liaoning Provincial Key Laboratory of Fine Separation Technique, University of Science and Technology, \\ Anshan 114051, Liaoning, China; askdwbf@163.com (B.W.); lnkdlsj@163.com (S.L.); \\ lnkdwym@163.com (Y.W.) \\ * Correspondence: aswsy64@163.com; Tel.: +86-412-592-9480
}

Academic Editor: Patrick van Rijn

Received: 27 April 2017; Accepted: 7 June 2017; Published: 9 June 2017

\begin{abstract}
The application of molecular imprinting technology in the separation and purification of active ingredients in natural products was widely reported, but remains a challenge. Enrichment and separation are especially limited. A surface imprinting technique was reported to synthesize molecularly imprinted microspheres (MIMs) in this article. With paeoniflorin (PF) as the template molecule, $\beta$-cyclodextrin ( $\beta$-CD) and acrylamide (AA) as the functional monomers, and poly (glycidyl methacrylate, GMA) microspheres $\left(\mathrm{P}_{\mathrm{GMA}}\right)$ as the backing material. MIMs have been characterized by FTIR and FESEM. Adsorption experiments indicated the adsorption capacity of MIMs was superior to those comparative non-imprinted microspheres (NIMs) and the binding isotherm of MIMs was in good agreement with the two-site binding model. The baseline separation of PF and its structural analogue albiflorin (AF) were achieved on the new MIMs packed column. MIMs showed good affinity and efficiency for separation of PF and AF compared with those comparative NIMs. The approach of fabricating MIMs is simple, rapid, and inexpensive, and may shed new light on the application of MIMs as a liquid chromatography stationary phase to separate and analyze PF and AF from the Red peony root extracts.
\end{abstract}

Keywords: molecular imprinting; stationary phase; paeoniflorin; chromatographic application

\section{Introduction}

Paeoniflorin (PF, CAS: 23180-57-6) is a monoterpene glycoside, which mainly exists in radix paeoniae rubra and radix paeoniae alba [1]. It has been declared to have antioxidant [2], anti-free radical damage [3], anti-platelet aggregation [4], improve microcirculation [5], and immune regulation [6]. It is relatively non-toxic and has served in cosmetics and medicines. In recent years, the separation of PF from herbs has attracted much attention in medical research. The traditional adsorbent, silica gel $[7,8]$, and macro-porous adsorption resin $[9,10]$ have been employed to separate and purify PF. However, the separation procedure is tedious and inefficient, and the traditional adsorbent is limited because of poor affinity and selectivity. It is difficult to enrich and separate PF using the traditional method because of the low content and the complex matrix in Chinese herbs. It is necessary to develop a more efficient method to enrich and separate PF.

Molecular imprinting technique is a rapidly developing technique for preparing highly cross-linked polymeric materials that contain highly specific recognition sites and with predetermined selectivity to template molecules [11]. Thus, the resulting molecularly imprinted polymers (MIPs) 
have the characteristics of the intended target, selectivity, and the recognition [12]. Because MIPs can selectively rebind the template or its analogs from a matrix, they have been extensively applied in solid-phase extraction (SPE) [13] and chromatography [14]. Because most MIPs have been synthesized by bulk polymerization and the preparation process is wasteful and time-consuming. The obtained irregularly shaped particles based on screening with MIPs were applied in SPE and high performance liquid chromatography (HPLC). Thus, more molecularly imprinted methods to obtain uniformly sized molecularly imprinted microspheres (MIMs) have been developed, such as seed polymerization [15], suspending polymerization [16], precipitation polymerization [17], and emulsion polymerization [18]. It is difficult to obtain MIMs with high affinity binding sites and selectivity with the preparation methods mentioned above, even those methods have significantly improved the adsorption properties of MIMs.

$\beta$-Cyclodextrin ( $\beta-C D)$ was a kind of cyclic oligosaccharide with a hollow cylindrical structure it has a hydrophilic exterior and an internal hydrophobic cavity [19]. The various kinds of inter-molecular interactions (van der Waals' force, hydrophobic interaction, electrostatic affinity, dipole-dipole interaction, and hydrogen bond interaction) exist in $\beta-C D$ and its derivatives, and of those interactions to entrap hydrophobic molecules. In recent years, $\beta-C D$ has been widely used in the preparation of MIPs [20,21]. Despite this, most $\beta$-CD MIPs are prepared by bulk polymerization; however, spherical $\beta$-CD MIPs have been reported [22].

In this paper, MIMs for PF were prepared by surface imprinting [19]. Uniformly sized poly (glycidyl methacrylate, GMA) microspheres $\left(\mathrm{P}_{\mathrm{GMA}}\right)$ were invoked as the backing material, $\beta-\mathrm{CD}$ and AA as the functional monomers, and PF as the template molecule. The performance of MIMs was evaluated by adsorption experiments and chromatographic experiments. The MIMs exhibited excellent PF chromatographic performance, such as good separation of PF and its structural analogue albiflorin (AF) and larger adsorption capacity. This research might provide a new approach to the further separation and purification of natural products from natural product extracts.

\section{Experimental}

\subsection{Instruments and Reagents}

Characterization of the MIMs were carried out using Fourier transformed infrared (FTIR) spectroscopy (Nicolet IS10, Thermo Fisher, Waltham, MA, USA). The morphologies and structures of the MIMs were characterized by field emission scanning electron microscopy (FESEM, operated at $5.0 \mathrm{kV}$, Oberkochen, Germany). LC-10AT system (Shimadzu, Kyoto, Japan) was used for chromatographic analyses. Chromatographic packing system (HY-HPLC-1, Beijing, China) was used for packing column with MIMs and NIMs. A V-sorb 2800P surface area and pore size analyzer (Gold APP Co., Beijing, China) was used to measure physical adsorption parameters.

$\mathrm{PF}$ and albiflorin (AF) (purity $>98 \%$ ) were obtained from Shenzhen ziker Biological Technology Co. Ltd. (Shenzhen, China). $\beta-\mathrm{CD}$ and sodium hydrogen $(\mathrm{NaH})$ were obtained from Tianjin Kermel Chemical Co. Ltd. (Tianjin, China). $\beta-\mathrm{CD}$ was recrystallized with distilled water before using. Structures of $\beta-C D, P F$, and AF are shown in Figure 1. Acrylamide (AA), styrene, PVP (K-30), and acetonitrile were purchased from Sinopharm Chemical Reagent Co. Ltd. (Shanghai, China). Ethyl glycol dimethacrylate (EGDMA), glycidyl methacrylate (GMA), poly(vinyl alcohol)-1788 (PVA-1788), and hydroxy ethyl methacrylate (HEMA) were obtained from Guangzhou Zhongye Chemical Co. Ltd. (Guangzhou, China). EGDMA was washed with 10\% sodium hydroxide solution, saturated sodium chloride solution, and washed with distilled water and then dried with anhydrous sodium sulfate before using. 2,2'-Azobisisobutyronitrile (AIBN) was purchased from Shanghai SHV Chemical Co. Ltd. (Shanghai, China) and was purified by recrystallization with ethanol before polymerization. The other used chemical reagent was of analytical grade. 


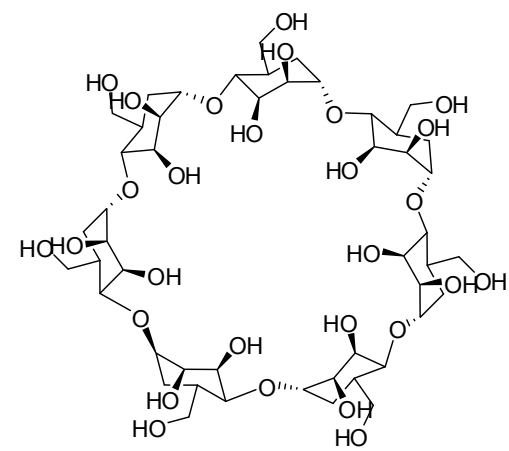

(a)<smiles>C[C@]12OC3OC1(OC1OC(CO)C(O)C(O)C1O)C1(OC(=O)c4ccccc4)CCC3(O)C12O</smiles>

(b)

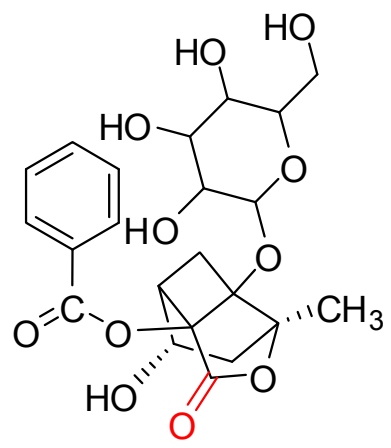

(c)

Figure 1. Structure of $\beta$-cyclodextrin $(\beta-C D)$, paeoniflorin $(P F)$, and albiflorin $(A F)$ : (a) $\beta$-CD; (b) PF; (c) AF.

\subsection{Synthesis of MIMs}

MIMS were prepared with $\beta-\mathrm{CD}$ and $\mathrm{AA}$, and $\mathrm{P}_{\mathrm{GMA}}$ were used as the support matrix. The scheme for preparation of MIMs is shown in Figure 2.

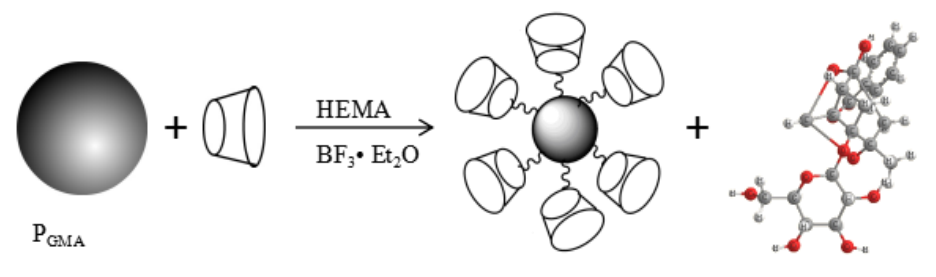

$\mathrm{P}_{\mathrm{GMA}}-\mathrm{CD}$

PF

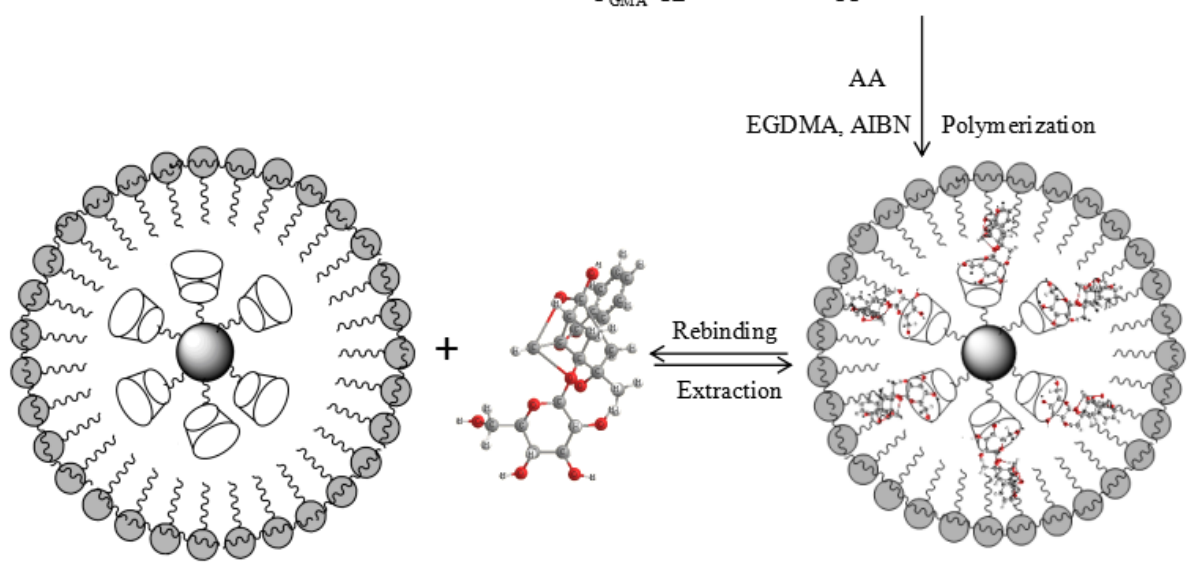

MIMs

Figure 2. Scheme for preparation of poly(glycidyl methacrylate) microspheres ( $\left.\mathrm{P}_{\mathrm{GMA}}\right)$-cyclodextrin (CD) and molecularly imprinted microspheres (MIMs).

\subsubsection{Synthesis of $\mathrm{P}_{\mathrm{GMA}}-\mathrm{CD}$}

Uniformly sized $\mathrm{P}_{\mathrm{GMA}}$ was prepared by the suspension polymerization method according to the reference [23].

The first step, $\mathrm{P}_{\mathrm{GMA}}-\mathrm{CD}$, was synthesized with $\beta$-CD and HEMA. In the process of preparation for $\mathrm{P}_{\mathrm{GMA}}-\mathrm{CD}, 6.72 \mathrm{~g}(6.0 \mathrm{mmol})$ of $\beta-\mathrm{CD}$ was dissolved in $120 \mathrm{~mL}$ of anhydrous dimethylformamide (DMF) to which $0.50 \mathrm{~g}$ of $\mathrm{NaH}$ was added. The mixture was stirred at $30^{\circ} \mathrm{C}$ in a water bath until no gas was emitted, and was filtrated to remove excessive $\mathrm{NaH}$. Seven grams of $\mathrm{P}_{\mathrm{GMA}}$ was added into the mixture, and the mixture was mechanically stirred at $30^{\circ} \mathrm{C}$ in a water bath for $4 \mathrm{~h}$. The solution 
temperature was increased by $10^{\circ} \mathrm{C}$ every $30 \mathrm{~min}$ until $80^{\circ} \mathrm{C}$; then, the solution was mechanically stirred at $80^{\circ} \mathrm{C}$ for $8 \mathrm{~h}$ under nitrogen protection.

In the second step, after the first reaction, the solution was naturally cooled to $40^{\circ} \mathrm{C}$, and a mixture solution containing $2.0 \mathrm{~g}$ of HEMA, $20 \mathrm{~mL}$ of DMF, and $40 \mu \mathrm{L}$ of $\mathrm{BF}_{3} \cdot \mathrm{Et}_{2} \mathrm{O}$ was added swiftly. The mixture was stirred at $40^{\circ} \mathrm{C}$ for $12 \mathrm{~h}$. The $\mathrm{P}_{\mathrm{GMA}}-\mathrm{CD}$ was obtained of $\beta-\mathrm{CD}$ and the vinyl group was bonded on the surface of microspheres. $\mathrm{P}_{\mathrm{GMA}} \mathrm{CD}$ microspheres were washed with DMF, methanol, distilled water, and acetone, and dried at $30^{\circ} \mathrm{C}$, respectively. The determination method of immobilized $\beta$-CD [24]: The immobilized $\beta$-CD support $\left(25.0 \mathrm{mg}\right.$ ) was hydrolyzed in $15 \mathrm{~mL}$ of $0.5 \mathrm{~mol} / \mathrm{L} \mathrm{H}_{2} \mathrm{SO}_{4}$ at $100{ }^{\circ} \mathrm{C}$ for $8 \mathrm{~h}$; then, the hydrolysate was made up to $50 \mathrm{~mL}$ with water, and the glucose content of hydrolysate was determined according to the method described in reference [25]. The amount of $\beta-C D$ bonded on PGMA can be calculated by the following formula:

$$
A_{(\beta-\mathrm{CD})}=\frac{C \times 50 \times 1000}{180 \times 7 \times W}=39.7 C / W
$$

where $A(\mu \mathrm{mol} / \mathrm{g})$ is the amount of $\beta-\mathrm{CD}$ bonded on $\mathrm{P}_{\mathrm{GMA}}, C(\mathrm{~g} / \mathrm{mL})$ is the glucose concentration in hydrolysate, and $W(\mathrm{mg})$ is the amount of immobilized $\beta$-CD support.

\subsubsection{Synthesis of MIMs}

The mixture containing $50.0 \mathrm{mg}(104.9 \mu \mathrm{mol})$ of PF, $65.0 \mathrm{mg}(914.5 \mu \mathrm{mol})$ of AA, $245 \mathrm{mg}(1.25 \mathrm{mmol})$ of EGDMA, $20 \mathrm{mg}$ of AIBN, and $50 \mathrm{~mL}$ of dimethylsulfoxide was added to a $100 \mathrm{~mL}$ three-neck flask that contained $3.0 \mathrm{~g}$ of $\mathrm{P}_{\mathrm{GMA}}-\mathrm{CD}$ microspheres. The mixture was stirred gently $(60 \mathrm{rpm})$ under nitrogen protection for $8 \mathrm{~h}$. Then, the flask was stirred $(600 \mathrm{rpm})$ in a water bath at $65^{\circ} \mathrm{C}$ for $8 \mathrm{~h}$ under constant nitrogen protection. The obtained MIMs were washed with distilled water and methanol to remove the template molecule and residual reagent.

Non-molecularly imprinted microspheres (NIMs) were prepared in the same way without the addition of PF for comparison.

Three kinds of MIMs and their comparative NIMs were prepared under different compositions, which are shown in Table 1.

Table 1. The preparative composition of different MIMs and non-imprinted microspheres (NIMs).

\begin{tabular}{cccccc}
\hline Polymer & PF $(\mu \mathrm{mol})$ & $\boldsymbol{\beta - C D}^{\mathbf{a}}(\boldsymbol{\mu m o l})$ & AA $(\boldsymbol{\mu m o l})$ & EGDMA (mmol) & AIBN $(\mathbf{m g})$ \\
\hline MIMs-1 & 104.9 & 102.9 & 914.5 & 1.25 & 20 \\
NIMs-1 & - & 102.9 & 914.5 & 1.25 & 20 \\
MIMs-2 & 104.9 & 102.9 & - & 1.25 & 20 \\
NIMs-2 & - & 102.9 & - & 1.25 & 20 \\
MIMs-3 & 104.9 & - & 914.5 & 1.25 & 20 \\
NIMs-3 & - & - & 914.5 & 1.25 & 20 \\
\hline \multicolumn{7}{c}{ a The content of bonded $\beta-C D}$.
\end{tabular}

\subsection{Adsorption Experiments}

Adsorption of PF from an aqueous solution ( $30 \%$ methanol) was investigated in batch experiments. The amount of MIMs, the adsorption isotherm, and the kinetics of the fabricated MIMs were determined. The adsorption capacity was calculated using the following equation:

$$
Q=\frac{\left(C_{0}-C_{\mathrm{e}}\right) \times V}{M}
$$

where $Q(\mathrm{mg} / \mathrm{g})$ is the amount of total adsorption of $\mathrm{PF}, C_{0}$ and $C_{\mathrm{e}}$ are the initial and equilibrium concentration of PF in solution $\left(\mathrm{mg} \cdot \mathrm{L}^{-1}\right)$, respectively, $V(\mathrm{~L})$ is the volume of the solution, and $M(\mathrm{~g})$ is the weight of the polymers. The imprinting factor (IF) was calculated according to IF $=Q_{\text {MIMs }} / Q_{\text {NIMs }}$. 
Fifty $\mathrm{mg}$ of MIMs and NIMs were placed in a tube and conditioned with $10 \mathrm{~mL}$ of $10 \mu \mathrm{mol} \cdot \mathrm{mL}^{-1}$ PF solutions ( $30 \%$ methanol). The tube was incubated in a shaken bed at $25^{\circ} \mathrm{C}$. The solutions were analyzed by HPLC at different time intervals, and each analysis was repeated three times.

Scathard analysis was used to estimate binding characteristics of MIMs-1 and NIMs-1 according to the Scathard equation:

$$
Q / C_{\mathrm{e}}=\frac{\left(Q_{\max }-Q\right)}{K_{\mathrm{D}}}
$$

where $K_{\mathrm{D}}$ is the equilibrium dissociation constant; $Q_{\max }$ is the apparent maximum number of binding sites; $C_{\mathrm{e}}$ is the equilibrium concentration of PF.

Fifty $\mathrm{mg}$ of MIMs or NIMs were placed in a tube and conditioned with $10 \mathrm{~mL}$ of $1.0-10 \mu \mathrm{mol} \cdot \mathrm{mL}^{-1}$ solutions of PF (each solution concentration increased by $1.0 \mu \mathrm{mol} \cdot \mathrm{mL}^{-1}$ ). The tube was incubated in a shaken bed at $25^{\circ} \mathrm{C}$ for $80 \mathrm{~min}$. The solutions were analyzed by HPLC, and each analysis was repeated three times.

For the polymers containing two types of binding sites, Scatchard analysis does not consider the contribution of the higher affinity binding sites and the lower affinity binding sites for the binding capacity of imprinted polymers at high concentrations and low concentrations of PF, respectively. Thus, the binding parameter values obtained by Scatchard analysis are inaccurate in the adsorption polymers that have two types of binding sites. The other equilibrium binding equation can be used to describe the adsorption process of two sites according to the following equation.

$$
Q=\frac{Q_{\max 1} C}{K_{d 1}+C}+\frac{Q_{\max 2} C}{K_{d 2}+C}
$$

where $Q$ is the amount of PF being adsorbed onto MIMs-1 or NIMs-1, $C$ is the free concentration of PF in solution, $Q_{\max 1}$ and $Q_{\max 2}$ are the apparent maximum adsorption capacity of the higher and lower affinity binding sites, respectively, and $K_{\mathrm{d} 1}$ and $K_{\mathrm{d} 2}$ are the corresponding equilibrium dissociation constants of MIMs-1. $Q^{\prime}{ }_{\max 1}$ and $Q^{\prime}{ }_{\max 2}$ are the apparent maximum adsorption capacity of the higher and lower affinity binding sites, respectively, and $K_{\mathrm{d} 1}^{\prime}$ and $K_{\mathrm{d} 2}^{\prime}$ are the corresponding equilibrium dissociation constants of NIMs-1.

Fifty $\mathrm{mg}$ of MIMs or NIMs were placed in a tube and conditioned with $10 \mathrm{~mL}$ of $1.0-10.0 \mu \mathrm{mol} \cdot \mathrm{mL}^{-1} \mathrm{PF}$ solutions (each solution concentration increased by $1.0 \mu \mathrm{mol} \cdot \mathrm{mL}^{-1}$ ). The tube was incubated in a shaken bed at $25^{\circ} \mathrm{C}$ for $80 \mathrm{~min}$. The solutions were analyzed by HPLC, and each analysis was repeated three times.

The selectivity of the fabricated MIMs and NIMs towards PF was studied by studying the adsorption of the MIMs and NIMs towards AF, the analogue of PF. $50 \mathrm{mg}$ of MIMs and NIMs were placed in a tube and conditioned with $10 \mathrm{~mL}$ of $10.0 \mu \mathrm{mol} \cdot \mathrm{mL}^{-1} \mathrm{PF}$ solutions and $10 \mathrm{~mL}$ of $10.0 \mu \mathrm{mol} \cdot \mathrm{mL}^{-1} \mathrm{AF}$ solutions. The tube was incubated in a shaken bed at $25^{\circ} \mathrm{C}$ for $80 \mathrm{~min}$. The solutions were analyzed by HPLC, and each analysis was repeated three times.

The analyses of PF were performed using the LC-10AT system. Chromatographic separations were performed on a Zorbax-ODS column $(150 \mathrm{~mm} \times 4.6 \mathrm{~mm}, 5 \mu \mathrm{m})$. The UV detection wavelength was $230 \mathrm{~nm}$. The mobile phase was a mixture of methanol-water-acetic acid (30:70:0.06, $v / v / v)$ and the flow rate was $1.0 \mathrm{~mL} \cdot \mathrm{min}^{-1}$. A calibration curve was obtained to facilitate quantitative determination of PF, which is $y=207.8808 x+4.9801\left(2.0-20.0 \mu \mathrm{mol} \cdot \mathrm{mL}^{-1}, R^{2}=0.9994\right)$.

\subsection{Chromatographic Experiments}

MIMs or NIMs were packed in a stainless steel column $(150 \mathrm{~mm} \times 4.6 \mathrm{~mm})$ by a dilute slurry column-packing method with HY-HPLC. The solvent of the packing column was isopropyl alcohol. The packed column was washed with the mobile phase at the flow rate of $0.3 \mathrm{~mL} \cdot \mathrm{min}^{-1}$ until the stable baseline was obtained. Analyses of MIMs and NIMs were performed with an LC-10AT system consisting of an LC-10ATvp and SPD-10A UV detector. Methanol of uracil was used to estimate 
the dead time $\left(t_{0}\right) . t_{\mathrm{R}}$ was the retention time, the retention factor $(k)$ was calculated according to $k=\left(t_{\mathrm{R}}-t_{0}\right) / t_{0}$, and the separation factor $(\alpha)$ was according to $\alpha=k_{\mathrm{PF}} / k_{\mathrm{AF}}$.

\section{Results and Discussion}

\subsection{Characterization of MIMs}

Uniformly sized MIMs were prepared by surface imprinting. The IR spectra of PGMA (a), $\beta$-CD (b), and MIMs-1 (c) are given in Figure 3. The typical feature of epoxy group around $918 \mathrm{~cm}^{-1}$ disappeared, and the typical feature intensity of $-\mathrm{CH}_{2}(\beta-\mathrm{CD})$ and $-\mathrm{OH}(\beta-\mathrm{CD})$ increased around 2930 $\mathrm{cm}^{-1}$ and around $3312 \mathrm{~cm}^{-1}$, respectively, based on the analysis for the main characteristic peak's position and intensity, which indicated that $\beta-C D$ was bonded on $P_{\mathrm{GMA}}$. The stretching vibration peak of $-\mathrm{C}-\mathrm{O}$ and $-\mathrm{C}-\mathrm{O}-\mathrm{C}$ around $1150 \mathrm{~cm}^{-1}$ and $1048 \mathrm{~cm}^{-1}$ were attributed to $\beta-\mathrm{CD}$ and $\beta-\mathrm{CD}$ polymers. The peak's positions around $1704 \mathrm{~cm}^{-1}$ and $1654 \mathrm{~cm}^{-1}$ were attributed to the carbonyl groups of polyacrylamide, which indicates the successful preparation of MIMs-1. According to the analysis of IR spectrum, it was proved that the hydrophilic groups (hydroxyl groups and hydrogen bonds) and hydrophobic groups (methyl, methylene, etc.) both produce affinity adsorption with PF, which indicates that the hydrophobic interaction and the hydrogen-bonding interaction exist simultaneously in MIMs-1.

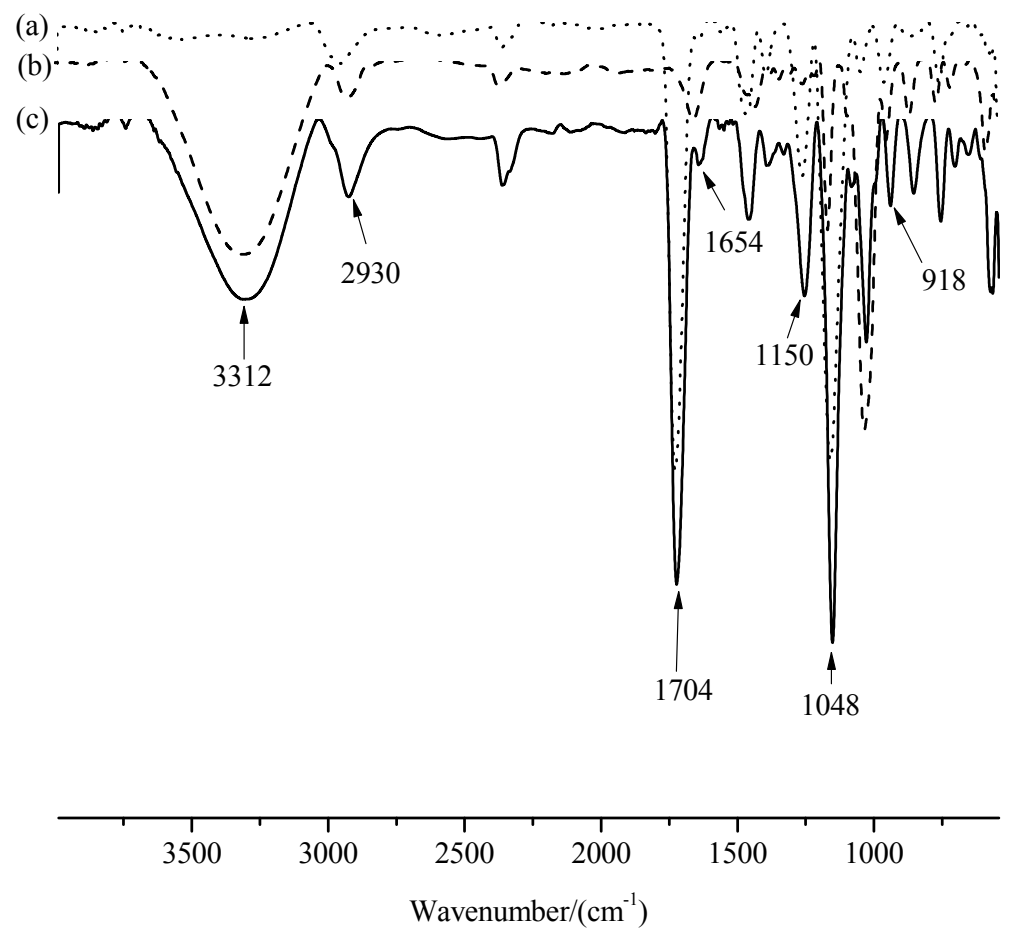

Figure 3. FTIR spectra of $\mathrm{P}_{\mathrm{GMA}}(\mathbf{a}), \beta-\mathrm{CD}(\mathbf{b})$, and MIMs-1 (c).

FESEM pictures of $\mathrm{P}_{\mathrm{GMA}}$ and MIMs-1 are shown in Figure 4. The diameters of $\mathrm{P}_{\mathrm{GMA}}$ were about $25 \mu \mathrm{m}$, and it can be seen that the surface was monoporous, but the diameters of MIMs were about $30 \mu \mathrm{m}$ and the surface was multi-porous. The change of the pore structure and the increase in diameter of the MIMs proved that $\beta-\mathrm{CD}$ and AA were bonded on the surface of $\mathrm{P}_{\mathrm{GMA}}$. A V-sorb 2800P surface area and pore size analyzer was used to measure the specific surface areas, the total pore volume, and the average pore diameters of three kinds of MIMs and their comparative NIMs by the nitrogen adsorption method. As shown in Table 2, the total pore volume, the average pore diameters, and the specific surface areas of MIMs and NIMs increased compared with $\mathrm{P}_{\mathrm{GMA}}$. The results showed that more complex $\beta-C D$ polymers were grafted on the surface of the $P_{\mathrm{GMA}}$ microspheres. The specific 
surface area of MIMs was less than that of NIMs, and the average pore diameter of MIMs was greater than that of NIMs, as shown in Table 2. Thus, compared to NIMs, MIMs showed a more regular pore structure and a more uniform pore size. The difference in specific pore volume between MIMs and NIMs was small. Thus, the template molecule had an indistinct impact on the specific pore volume.

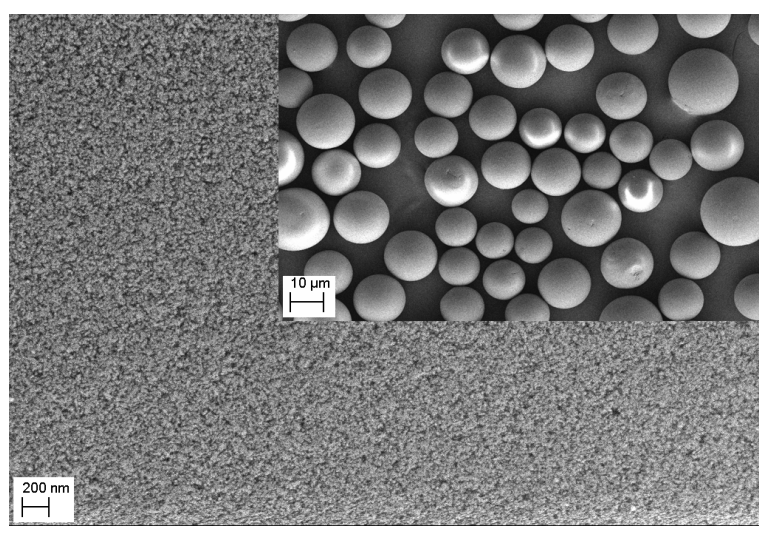

(a)

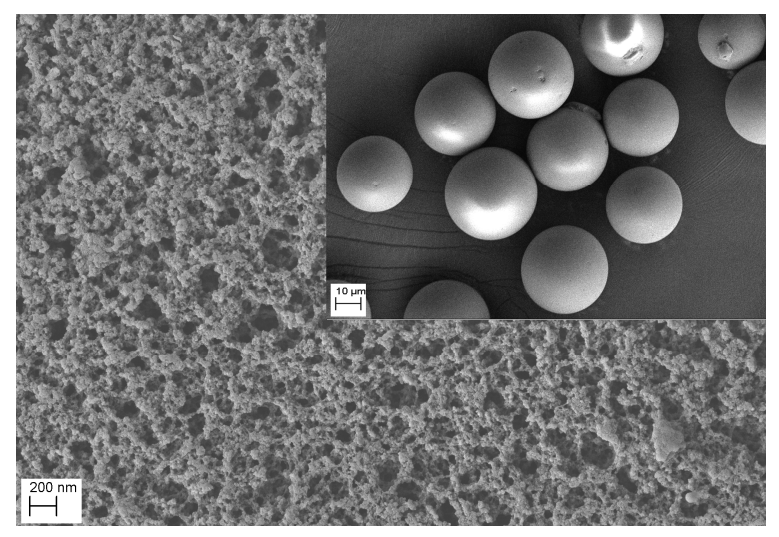

(b)

Figure 4. Scanning electron micrographs of $\mathrm{P}_{\mathrm{GMA}}(\mathbf{a})$ and MIMs-1 (b).

Table 2. Properties of $\mathrm{P}_{\mathrm{GMA}}$, MIMs, and NIMs.

\begin{tabular}{cccc}
\hline Polymer & $\begin{array}{c}\text { Specific surface area } \\
\left(\mathbf{m}^{\mathbf{2}} \cdot \mathbf{g}^{\mathbf{~}} \mathbf{)}\right.\end{array}$ & $\begin{array}{c}\text { Average pore diameter } \\
\mathbf{b}\end{array}$ & $\begin{array}{c}\text { Specific pore volume }^{\mathbf{c}} \\
(\mathbf{m} \mathbf{m})\end{array}$ \\
\hline $\mathrm{P}_{\mathrm{GMA}} \mathbf{g}^{\mathbf{- 1}} \mathbf{)}$
\end{tabular}

a Measured by Brunauer-Emmett-Teller (BET) method; ${ }^{\text {b }}$ Measured by Dubinin-Radushkevich (D-R) method;

${ }^{\mathrm{c}}$ Measured by the Barrett-Joyner-Halenda (BJH) method.

\subsection{Adsorption Studies of MIMs}

The adsorption kinetic curves of PF on MIMs and NIMs are shown in Figure 5. The figure shows that the adsorption capacity of MIMs for PF was greater than that of the corresponding NIMs, and the adsorption capacity of MIMs-1 for PF was batter than that of MIMs-2 and MIMs-3. In addition, the adsorption equilibrium was achieved quickly. The main cause of the results was $\mathrm{P}_{\mathrm{GMA}}$ was monoporous microspheres similar to silica microspheres, so the imprinted recognition sites were mainly bonded on the surface of MIMs. The IF of these three kinds of MIMs is shown in Table 3. The differences in IF revealed that MIMs- 1 had a higher affinity when bonding with both $\beta-C D$ and AA than the other two imprinted polymers microspheres when bonding with $\beta-\mathrm{CD}$ and AA separately; therefore, the hydrophobic interaction and the hydrogen-bonding interaction exist simultaneously in MIMs-1. This indicated that the structures of MIMs were different from NIMs because the structures of template molecules preserved in the MIMs and NIMs do not have the template structures, and the differences in the IF are mainly due to the "memory" recognition sites. Because $\beta$-CD has a hydrophilic exterior and an internal hydrophobic cavity, MIMs-1 has an inclusion effect on paeoniflorin, except the hydrophobic interaction and the hydrogen-bonding interaction. However, MIMs-3 and NIMs-3 have no inclusion effect on paeoniflorin, and the preparation methods of MIMs-2 (and NIMs-2) and MIMs-3 (and NIMs-3) were the traditional preparation methods of molecularly imprinted polymers with only one interaction. These results indicate a hydrophobic interaction combined with a hydrogen-bonding 
interaction, and the inclusion effect was stronger than both the hydrophobic interaction and the hydrogen-bonding interaction. Therefore, in the following studies, only MIMs-1 was investigated.

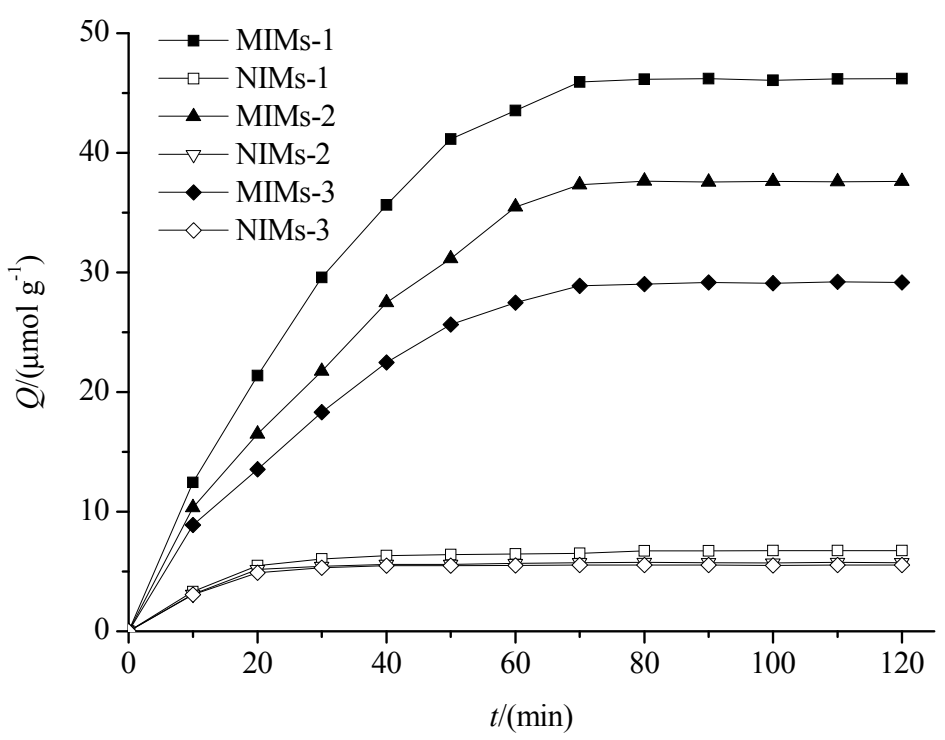

Figure 5. The adsorption dynamics curves of the MIMs and NIMs. Adsorption conditions: $10 \mathrm{~mL}$ of $10 \mu \mathrm{mol} \cdot \mathrm{mL}^{-1}$ solutions (30\% methanol) of PF with $50 \mathrm{mg}$ of MIMs or NIMs.

Table 3. Imprinting factor (IF) of three kinds of MIMs.

\begin{tabular}{ccc}
\hline Polymer & $\mathbf{Q}^{\mathbf{a}}$ & IF \\
\hline MIMs-1 & 45.93 & 7.06 \\
NIMs-1 & 6.51 & \\
MIMs-2 & 37.33 & 6.53 \\
NIMs-2 & 5.72 & \\
MIMs-3 & 28.87 & 5.22 \\
NIMs-3 & 5.53 & \\
\cline { 2 - 3 } a The amount of total adsorption of PF were measured at $70 \mathrm{~min}$.
\end{tabular}

The Scatchard dissociation curves of PF on MIMs-1 and NIMs-1 are shown in Figure 6. There were two discontinuous liners for MIMs-1, which indicated there were two types of affinity binding sites in MIMs-1. On the contrary, there was only one line for NIMs-1, which indicated there was one type of binding sites in NIMs-1. For MIMs-1, the regression equation was $Q / C_{\mathrm{e}}=-1.300 Q+66.20$ $(\mathrm{r}=0.9816)$ for the higher affinity binding site, and the regression equation was $Q / C_{\mathrm{e}}=-0.148 Q+$ $30.61(\mathrm{r}=0.9574)$ for the lower affinity binding site. Therefore, $K_{\mathrm{D}}$ and $Q_{\max }$ were $0.7692 \mathrm{mmol} \cdot \mathrm{g}^{-1}$ and $50.92 \mu \mathrm{mol} \cdot \mathrm{g}^{-1}$ for the higher affinity binding site, and $6.7568 \mathrm{mmol} \cdot \mathrm{g}^{-1}$ and $206.83 \mu \mathrm{mol} \cdot \mathrm{g}^{-1}$ for the lower affinity binding site, respectively, according to the two regression lines. For NIMs-1, the regression equation was $Q / C_{\mathrm{e}}=-0.626 Q+5.9953(\mathrm{r}=0.9831)$, and $K_{\mathrm{D}}$ and $\mathrm{Q}_{\max }$ were $1.5974 \mathrm{mmol} \cdot \mathrm{g}^{-1}$ and $9.577 \mu \mathrm{mol} \cdot \mathrm{g}^{-1}$, respectively. The main reason for the difference of the Scatchard curves of MIMs-1 and NIMs- 1 were the different recognition sites were preserved in the two kinds of polymers.

Meanwhile, the equilibrium binding fitting curve of PF on MIMs and NIMs was obtained by $Q$ versus $C$ according to Equation (4) and is shown in Figure 7. As shown in Figure 7, the fitting curve was in good agreement with the experimental points. For MIMs, $Q_{\max 1}, Q_{\max 2}, K_{\mathrm{d} 1}$, and $K_{\mathrm{d} 2}$ were $16.46 \mu \mathrm{mol} \cdot \mathrm{g}^{-1}, 595.85 \mu \mathrm{mol} \cdot \mathrm{g}^{-1}, 0.2453 \mathrm{mmol} \cdot \mathrm{L}^{-1}$, and $36.98 \mathrm{mmol} \cdot \mathrm{L}^{-1}$, and the correlation coefficient of the fitting curve was 0.9975 . Moreover, for NIMs, $Q^{\prime}{ }_{\max 1}, Q^{\prime}{ }_{\max 2}, K_{\mathrm{d} 1}^{\prime}$, and $K_{\mathrm{d} 2}^{\prime}$ were $169.84 \mu \mathrm{mol} \cdot \mathrm{g}^{-1}, 7.21 \mu \mathrm{mol} \cdot \mathrm{g}^{-1}, 39.81 \mathrm{mmol} \cdot \mathrm{L}^{-1}$, and $1.26 \mathrm{mmol} \cdot \mathrm{L}^{-1}$, and the correlation coefficient of the fitting curve was 0.9997 . The difference between $Q_{\max }$ and $K_{\mathrm{d}}$ indicated that the MIMs have a higher selective adsorption capacity $\left(Q_{\max 2}>Q_{\max 2}^{\prime}\right)$ than NIMs, and NIMs have a higher non-selective 
adsorption capacity $\left(Q_{\max 1}<Q_{\max 1}^{\prime}\right)$ than MIMs. This indicates that the values obtained by the equilibrium binding equation are more workable compared to the Scathard analysis.
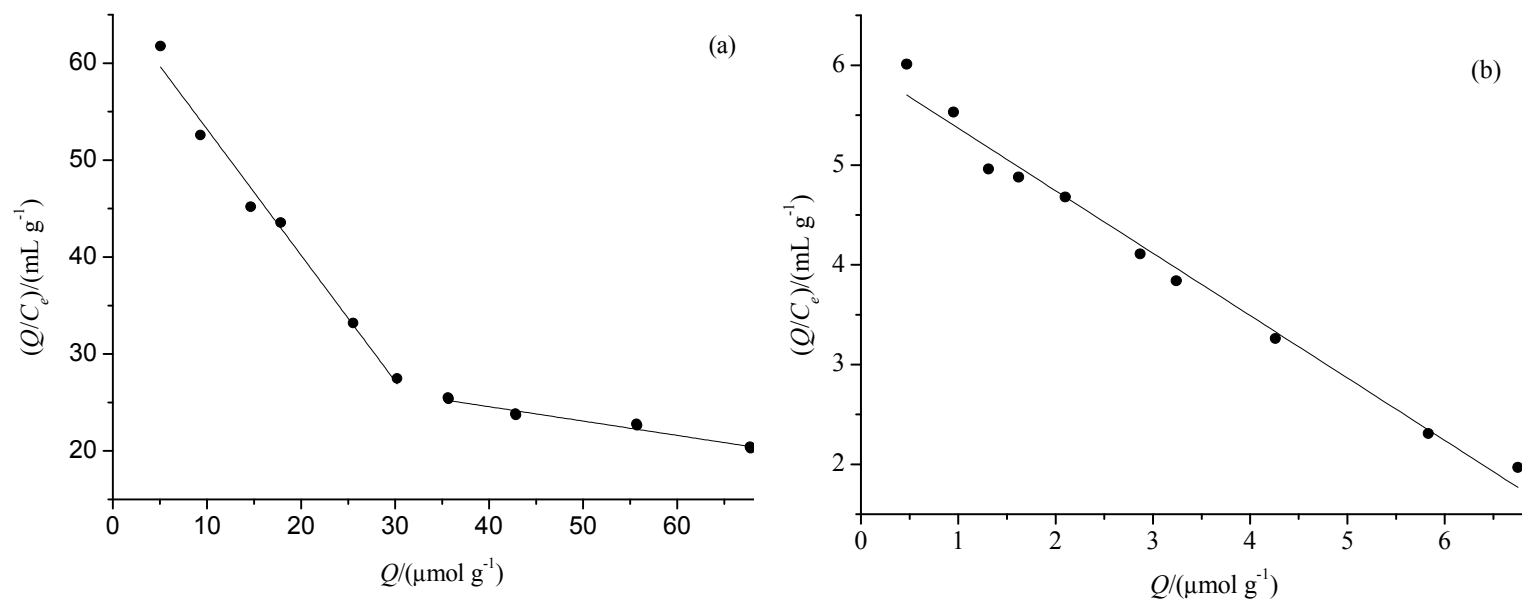

Figure 6. The Scatchard plots of MIMs-1 (a) and NIMs-1 (b) for PF.

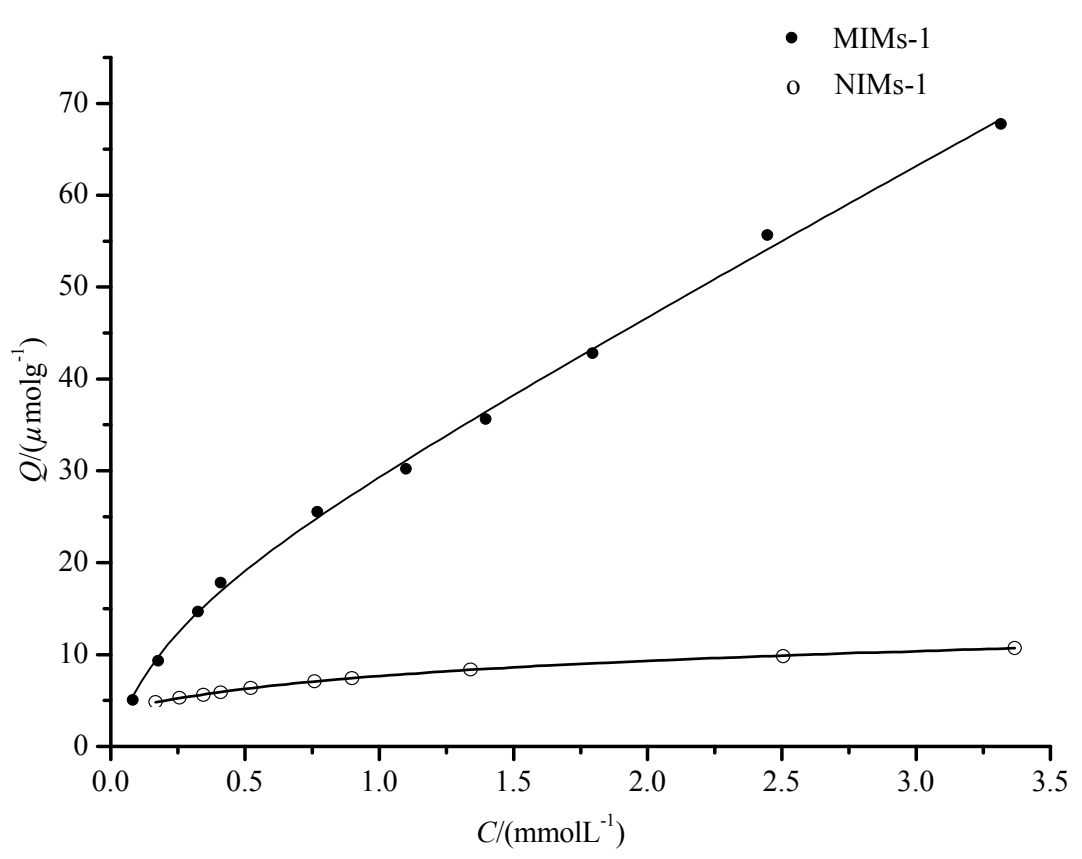

Figure 7. The fitting curve of equilibrium binding equation.

Selectivity of MIMs with regard to NIMs was studied, and the result is shown in Figure 8. As can be seen from Figure 8, MIM particles have a higher selective adsorption towards PF compared to AF, while the adsorption capacities of PF and AF by NIMs were almost similar to each other. Imprinting sites formed in the MIMs have the capability of distinguishing target molecules through their size, shape, and functional group distribution. However, there are non-imprinting sites in NIMs to adsorb $\mathrm{PF}$ and $\mathrm{AF}$. 


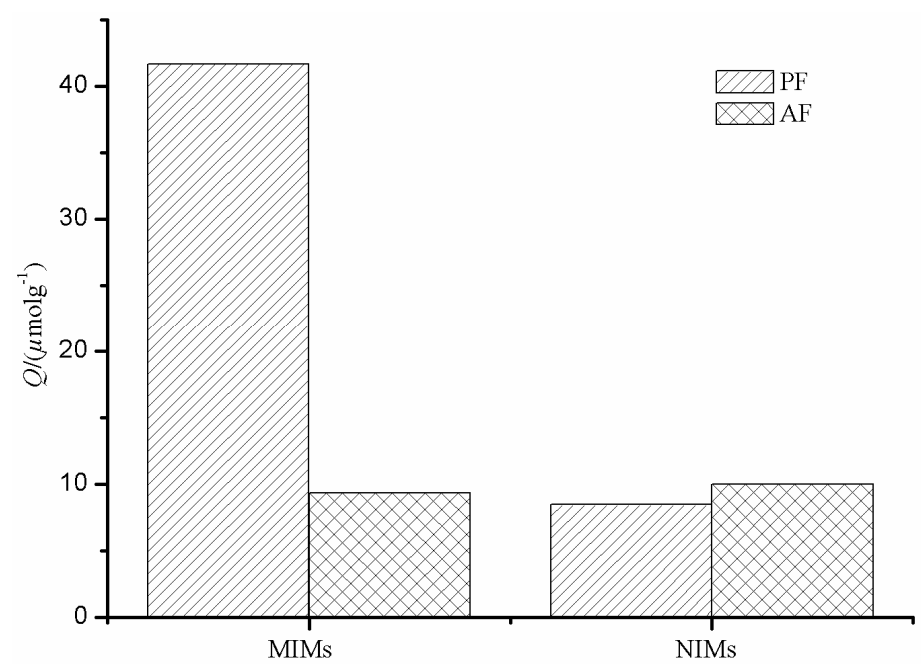

Figure 8. Selectivity study of PF and AF with MIMs and NIMs.

\subsection{Chromatographic Analysis}

PF and its similar structure AF have analogous properties, and they are all monoterpene glycosides. Therefore, it is difficult to separate PF and AF using the traditional column chromatography. The feasibility of utilizing MIMs- 1 as HPLC stationary phase for the separation of PF and AF was investigated. The chromatogram of PF and AF on the reversed phase columns with MIMs-1 and NIMs-1 as the stationary phase (as shown in Section 2.4), respectively, is shown in Figure 9a. As shown in Figure 9a, baseline separation was achieved on the imprinted column of PF and AF, and it could not be achieved on the non-imprinted column. The separation factor was 1.47 on the imprinted column. The main cause of the difference in separation on the two columns was the special recognition sites existing in the MIMs-1.
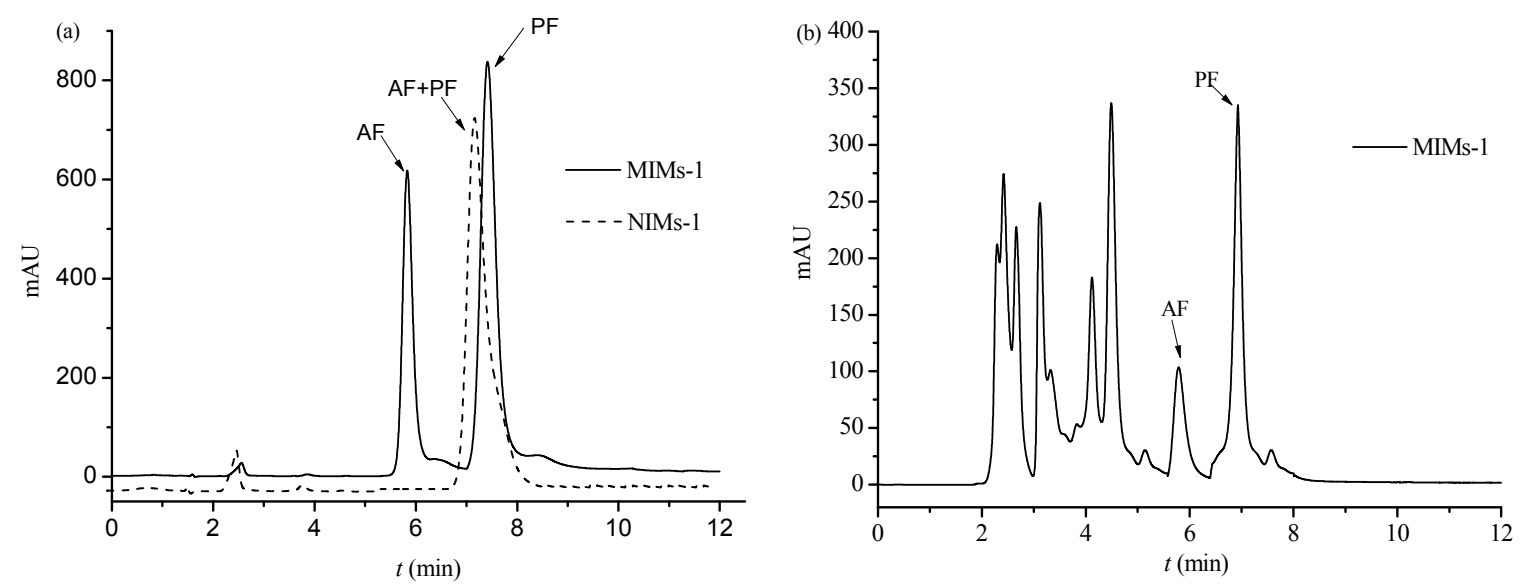

Figure 9. Chromatograms of PF and AF on MIMs-1/NIMs-1 (a) and Chromatogram of Red peony root extracts on MIMs-1 (b). LC conditions of MIMs-1/NIMs-1 (a,b): mobile phase: methanol-water-acetic acid $(27: 73: 0.06, v / v / v)$; flow rate was $0.5 \mathrm{~mL} \cdot \mathrm{min}^{-1}$; extracting conditions of Red peony root extracts: $10 \mathrm{~g}$ of Red peony root powder was dissolved in $40 \mathrm{~mL}$ of $50 \%$ ethanol solution (v \%), the extraction temperature was $50{ }^{\circ} \mathrm{C}$, and the time was $3 \mathrm{~h}$.

Generally speaking, the performance of the MIMs was strongly dependent on the solvent/eluent used in the adsorption/desorption processes, especially for non-specific interactions. The methanol/ water mixtures were specifically selected in the testing of the materials after the experimental optimization (see [26]). The results indicate that the viscosity of ethanol was greater than that of 
methanol and acetonitrile at room temperature. Furthermore, the viscosity of ethanol was similar to that of methanol and acetonitrile at $50^{\circ} \mathrm{C}$. However, chromatographic separation is usually performed at room temperature. In addition, acetonitrile is expensive, and its elution capacity is similar to that of methanol. Therefore, methanol was chosen as solvent/eluent. The choice of methanol/water ratio was mainly the optimization result of chromatographic separation and retention time.

More complex components exist in the Red peony root extracts, except PF and AF. Therefore, it is more difficult to separate PF from the Red peony root extracts. The chromatogram of the Red peony root extracts on the imprinted column (as shown in Section 2.4) is shown in Figure 9b. It can be seen that there were many different constituents in the extracts. PF and AF could also be separated by baseline. These results indicate the possibility of applying MIMs-1 as the stationary phase to analyze or separate the Red peony root extracts.

\section{Conclusions}

To separate PF and AF, a new approach for the fabrication of the PF MIMs was presented by molecular imprinting. The modified MIMs exhibited a larger adsorption capacity $\left(206.83 \mu \mathrm{mol} \cdot \mathrm{g}^{-1}\right)$, good recognition, and a good chromatographic separation performance. Compared with MIMs-2 and MIMs-3, which have single affinity binding sites, the MIMs-1, which has two types of affinity binding sites, has a higher affinity and selectivity for $\mathrm{PF}$, and the adsorption isotherm is in good agreement with the two-site binding model. PF and AF can be separated at baseline on the imprinted column, with MIMs-1 as the stationary phase. The MIMs-1 could act as an adsorbent for separating Red peony root extracts. In addition, this fabrication procedure is simple, rapid, and inexpensive. We believe that this method may inform the separation of other natural product intermediates that have an analogue structure.

Acknowledgments: The research was supported by the Education Department Foundation of Liaoning Province, China (No. 2016HZPY03), (No. L2014106) and the National Natural Science Foundation of China (No. 21576127).

Author Contributions: Shaoyan Wang and Wei Zhang conceived and designed the experiments; Yueming Wang performed the experiments; Wei Zhang, Shoujiang Li, and Shaoyan Wang analyzed the data; Bofeng Wei contributed reagents/materials/analysis tools; Wei Zhang wrote the paper.

Conflicts of Interest: The authors declare no conflict of interest.

\section{References}

1. Kaneda, M.; Iitaka, Y.; Shibata, S. Chemical studies on the oriental plant drugs-XXXIII: The absolute structures of paeoniflorin, albiflorin, oxypaeoniflorin and benzoylpaeoniflorin isolated from Chinese paeony root. Tetrahedron 1972, 28, 4309-4317. [CrossRef]

2. Zhang, M.H.; Feng, L.; Zhu, M.M.; Gu, J.F.; Wu, C.; Jia, X.B. Antioxidative and anti-inflammatory activities of paeoniflorin and oxypaeoniflora on AGEs-induced mesangial cell damage. Planta Med. 2013, 79, 1319-1323. [CrossRef] [PubMed]

3. Sun, R.; Yi, Y.P.; Lv, L.L.; Zhang, Z.P.; Sun, H.; Liu, G.Q. Effects of paeoniflorin on pathological changes in global brain ischemia model rats. China J. Chin. Mater. Med. 2007, 32, 2518-2522.

4. Tang, X.; Liu, J.; Dong, W.; Li, P.; Li, Lei.; Lin, C.R.; Zheng, Y.Q.; Hou, J.C.; Li, D. The cardioprotective effects of citric acid and L-malic acid on myocardial ischemia/reperfusion injury. Evid. Based Complement. Altern. Med. 2013, 3, 820695. [CrossRef] [PubMed]

5. Rosen, R. Herbal Skin Formulation. U.S. Patent 7,235,265, 26 June 2007.

6. Yoshihisa, Y.; Furuichi, M.; Rehman, M.R.; Ueda, C.; Makino, T.; Shimizu, T. The Traditional Japanese Formula Keishibukuryogan Inhibits the Production of Inflammatory Cytokines by Dermal Endothelial Cells. Mediat. Inflamm. 2010, 1, 60-68. [CrossRef] [PubMed]

7. Liu, A.L.; Zhang, S.; Wang, K.B.; He, W.; Liu, H.Y.; He, Y.C. Purification technology of paeoniflorin and albiflorin from Paeonia lactiflora Pall. J. Tradit. Chin. Med. Univ. Hunan 2008, 3, 35-37.

8. Shibutani, S.; Nagasawa, T.; Oura, H.; Nonaka, G.; Nishioka, I. Effect of extract from Paeoniae Radix on urea-nitrogen concentration in rat serum. I. Chem. Pharm. Bull. 1981, 29, 874-878. [CrossRef] [PubMed] 
9. Dou, Z.H.; Luo, L.; Lu, D. Purification of total paeony glycoside by macroporous resin with double indices of albiflorin and paeoniflorin. J. Chin. Med. Mater. 2009, 32, 1282-1284.

10. Qin, A.X.; Shu-Jing, J.I.; Mao, W.Y.; Gao, Z.; Kang, X.H.; Jia, Y.N.; Chen, C.; Cui, T. Identification and Purification of Paeoniflorins in Seed Cakes of Paeonia sufruticosa by Macroporous Adsorption Resin. Prepr. Pap. Am. Chem. Soc. Div. Fuel Chem. 1989, 34, 63-66.

11. Wullff, G. Molecular recognition in polymers prepared by imprinting with templates. Am. Chem. Soc. 1986, 9, 186-230.

12. Tan, C.J.; Tong, Y.W. Molecularly imprinted beads by surface imprinting. Anal. Bioanal. Chem. 2007, $389,369$. [CrossRef] [PubMed]

13. Caro, E.; Marcé, R.M.; Cormack, P.A.; Sherrington, D.C.; Borrull, F. Molecularly imprinted solid-phase extraction of naphthalene sulfonates from water. J. Chromatogr. A 2004, 1047, 175-180. [CrossRef]

14. Andrade, F.N.; Santosneto, A.J.; Lanças, F.M. Microextraction by packed sorbent liquid chromatography with time-of-flight mass spectrometry of triazines employing a molecularly imprinted polymer. J. Sep. Sci. 2014, 37, 3150-3156. [CrossRef] [PubMed]

15. Chen, Z.; Zhao, R.; Shangguan, D.; Liu, G.Q. Preparation and evaluation of uniform-sized molecularly imprinted polymer beads used for the separation of sulfamethazine. Biomed. Chromatogr. 2005, 19, 533-538. [CrossRef] [PubMed]

16. Lai, J.P.; Lu, X.Y.; Lu, C.Y.; Ju, H.F.; He, X.W. Preparation and evaluation of molecularly imprinted polymeric microspheres by aqueous suspension polymerization for use as a high-performance liquid chromatography stationary phase. Anal. Chim. Acta 2001, 442, 105-111. [CrossRef]

17. Shi, Y. Narrowly dispersed molecularly imprinted microspheres prepared by a modified precipitation polymerization method. Anal. Chim. Acta 2008, 612, 105.

18. Lu, S.; Cheng, G.; Zhang, H.; Pang, X. Preparation and characteristics of Tryptophan-imprinted $\mathrm{Fe}_{3} \mathrm{O}_{4} / \mathrm{P}$ (TRIM) composite microspheres with magnetic susceptibility by inverse emulsion-suspension polymerization. J. Appl. Polym. Sci. 2006, 99, 3241-3250. [CrossRef]

19. Liu, H.M.; Liu, C.H.; Yang, X.J.; Zeng, S.J.; Xiong, Y.Q.; Xu, W.J. Uniformly sized $\beta$-cyclodextrin molecularly imprinted microspheres prepared by a novel surface imprinting technique for ursolic acid. Anal. Chim. Acta 2008, 628, 87-94. [CrossRef]

20. Suzuki, I.; Egawa, Y.; Mizukawa, Y.; Hoshi, T.; Anzai, J. Construction of positively-charged layered assemblies assisted by cyclodextrin complexation. Chem. Commun. 2002, 13, 164. [CrossRef]

21. Pariot, N.; Edwards-Lévy, F.; Andry, M.C.; Lévy, M.C. Cross-linked $\beta$-cyclodextrin microcapsules. II. Retarding effect on drug release through semi-permeable membranes. Int. J. Pharm. 2002, 232, 175-181. [CrossRef]

22. Pariot, N.; Edwards-lévy, F.; Andry, M.C.; Lévy, M.C. Cross-linked beta-cyclodextrin microcapsules: preparation and properties. Int. J. Pharm. 2000, 211, 19. [CrossRef]

23. Gong, B.; Shen, Y.; Geng, X. Preparation of Strong Cation Exchange Packings Based on Monodisperse Poly(glycidyl methacrylate-co-ethylenedimethacrylate) Particles and Their Application. J. Liq. Chromatogr. Relat. Technol. 2003, 26, 963-976. [CrossRef]

24. He, B.; Zhao, X. Study on the Synthesis and Characterization of Novel Immobilized $\beta$-Cyclodextrin Polymer (I). Sci. China Ser. B 1993, 7, 19-29.

25. Dubois, M.; Gilles, K.A.; Hamilton, J.K.; Rebers, P.; Smith, F. Colorimetric Method for Determination of Sugars and Related Substances. Anal. Chem. 1956, 28, 350-356. [CrossRef]

26. Fu, C.; Li, Z.; Liu, S.; Chen, C. The study of Ethanol Water Used as Reversed Phase High Performance Liquid Chromatographic Mobile Phase. Chin. J. Chromatogr. 1999, 17, 466-468.

(C) 2017 by the authors. Licensee MDPI, Basel, Switzerland. This article is an open access article distributed under the terms and conditions of the Creative Commons Attribution (CC BY) license (http:/ / creativecommons.org/licenses/by/4.0/). 